\title{
Determination of Multiple Sclerosis Indicators for Value-Based Contracting Using the Delphi Method
}

\author{
Elizabeth C.S. Swart, BS; Lynn M. Neilson, PhD; Chester B. Good, MD, MPH; \\ William H. Shrank, MD, MSHS; Rochelle Henderson, PhD; Chronis Manolis, RPh; \\ and Natasha Parekh, MD, MS
}

\begin{abstract}
BACKGROUND: Value-based contracts link medication payments to performance measures with the ultimate goal of lowering costs while improving patient outcomes. Previous multiple sclerosis (MS) value-based contracts have focused on indicators easily collected from claims or electronic health record data as their value-based outcomes, even though numerous other MS clinical indicators of interest exist. Uncertainty remains regarding which MS indicators are most meaningful to all stakeholders affected by a value-based contract.
\end{abstract}

OBJECTIVE: To identify meaningful MS indicators among key stakeholders for the purpose of informing a value-based contract for MS medications.

METHODS: Using a modified Delphi method, we surveyed 26 diverse stakeholders, including 8 patients and caregivers; 9 providers (neurologists, nurses, physician assistants, and specialty pharmacists); 2 pharmaceutical company representatives; 5 payers; and 2 pharmacy benefits managers. A list of 12 MS indicators was created from subject matter expert consultation and a literature review. All stakeholders reported on the meaningfulness and value of these 12 indicators through a 5-point Likert scale and forced selection of the 3 most meaningful indicators. All nonpatient stakeholders were additionally surveyed on collection feasibility of the same 12 indicators using a 5-point Likert scale. We defined consensus as $\geq 75 \%$ agreement on the meaningfulness and feasibility of an indicator (Likert scores 4 or 5). We performed a Fisher's exact test to assess differences between nonpatient and patient stakeholder rankings of indicators.

RESULTS: Consensus was reached for at least 1 indicator for all questions after 2 rounds. "Worsening physical disability" and "functional impairment" achieved $92 \%$ agreement on a Likert-scale question assessing indicator value, and $100 \%$ of participants selected "worsening physical disability" when asked to choose the 3 most meaningful indicators. "MS flares requiring an emergency department visit" and "MS flares requiring inpatient admission" were rated as the 2 most feasibly collected indicators (both received $89 \%$ agreement).

CONCLUSIONS: Using the Delphi method, we identified that disability and functional impairment are meaningful MS indicators to diverse stakeholders. These findings support the incorporation of important patient-reported outcomes into value-based contracts for MS medications.

J Manag Care Spec Pharm. 2019;25(7):753-60

Copyright @ 2019, Academy of Managed Care Pharmacy. All rights reserved.

\section{What is already known about this subject}

The costs of disease-modifying therapies and other multiple sclerosis (MS) medications continue to rise at rates 5-7 times more than inflation, making them one of the highest-expenditure drug classes.

Previous value-based contracts for MS medications have connected payment to outcome indicators derived from claims and electronic health record data, including MS-related emergency department visits, hospitalizations, medication adherence, and relapse rates.

Selection of clinical indicators that are meaningful to all stakeholders and feasibly collected remains a challenge in the development of effective MS value-based contracts.

\section{What this study adds}

A stakeholder panel of patients, payers, providers, and pharmaceutical company and pharmacy benefits manager representatives identified meaningful MS indicators to inform a value-based contract for MS medications.

Stakeholders rated on a Likert scale "worsening physical disability" and "functional impairment" as the most valuable indicators for providing information about the status of MS.

"MS flares requiring an emergency department visit" and "MS flares requiring inpatient admission" were identified as the 2 most feasibly collected MS indicators by nonpatient stakeholders.

$\mathrm{M}$ ultiple sclerosis (MS) is a progressive autoimmune disease of the central nervous system that currently affects between 400,000 and 570,000 people in the United States and an estimated 2.5 million people globally.,2 Management of MS is inherently complex and is further complicated by the fact that many individuals are diagnosed between ages 20 and 50 years. ${ }^{3}$ This diagnosis can greatly diminish one's income-earning ability and impose an extreme financial burden on patients and families. ${ }^{4}$ Costs stemming from MS cascade into the health care system and society, with annual spending at an estimated $\$ 2.5$ billion in the United States alone. ${ }^{5}$

Disease-modifying therapies to treat MS are among the most expensive drug classes in the country. ${ }^{6} \mathrm{~A}$ recent analysis 
reported that costs of most disease-modifying therapies exceed $\$ 70,000$ a year. ${ }^{5}$ Furthermore, costs are increasing at rates 5 to 7 times more than inflation. ${ }^{7}$ In response to the rising costs of specialty medications such as disease-modifying therapies, there is increased interest in developing value-based contracts. Here, payers and manufacturers are incentivized to agree on tying outcome-based indicators with reimbursement for medications, in contrast with conventional payer-manufacturer contracts that are based on volume of medications used and willingness to pay. ${ }^{8-10}$

Because numerous MS clinical indicators of interest exist and there are challenges in measuring some indicators via pharmacy and administrative claims data, uncertainty remains regarding which MS indicators should be included in valuebased contracts. This uncertainty has been further perpetuated by opacity in current value-based contracts in the market that prohibit meaningful shared learning about how to best develop and execute value-based contracts. The few MS value-based contracts that have been documented in the marketplace have primarily focused on MS relapses, emergency room visits, and hospitalizations as their value-based outcomes, but it is unclear whether these are meaningful to all stakeholders affected by a value-based contract. ${ }^{10}$

In this study, we sought to incorporate multiple stakeholder perspectives to transparently identify relevant and measurable MS clinical indicators for the specific purpose of informing a meaningful value-based contract through use of the Delphi method.

\section{Methods}

The Delphi method is a structured, iterative survey method that is uniquely positioned to facilitate consensus building among diverse stakeholders. This method has been effectively employed to respond to a multitude of health care inquiries, such as prescribing standards and disease-specific treatment plans. ${ }^{11,12}$ We used our modified Delphi survey to seek consensus on indicators for a value-based contract among relevant stakeholders consisting of patients/caregivers, providers, payers, and pharmaceutical company and pharmacy benefits manager representatives. ${ }^{13,14}$ This technique allowed us to anonymously leverage our stakeholders' varied experiences, most notably those of patients and providers, to better understand which MS indicators are most meaningful. Traditional Delphi methodology begins with an open-ended round to collect a broad list of measures from panelists; we opted to perform a modified Delphi method by providing an established list of common MS clinical indicators in the first survey round and allowing panelists to suggest additional indicators for future rounds. ${ }^{14}$ This study was submitted to the University of Pittsburgh Institutional Review Board and was deemed exempt because the survey procedure was identified as having low risk of harm and responses were deidentified.

\section{Survey Design}

We selected 12 indicators for our Round 1 modified Delphi survey (Appendix, available in online article) through a multistep process. A pilot survey was developed by compiling an initial list of indicators from a review of disability scales currently used to monitor MS (i.e., Expanded Disability Status Score, Scripps Neurological Rating Scale, MS Impact Scale-29, and MS Quality of Life-54 $4^{15-23}$ ) and the Consensus Criteria for Sub-Optimal Response, ${ }^{24}$ a guide developed by neurologists to evaluate treatment response and disease progression. The first version of the pilot survey included 13 indicators and was reviewed by 6 UPMC Health Plan MS care managers, 5 pharmacists, 3 primary care physicians, and 1 neurologist specializing in MS to ensure that a wide range of opinions were obtained. Based on the pilot feedback, medication adherence was removed as an indicator option because it is difficult to define what constitutes adherence in MS, since providers often reduce medication doses and frequency due to side effects, efficacy, and costs. Additionally, some indicators were reworded for clarity and comprehensibility, and plain language definitions were added for each indicator. The final survey version achieved face validity among this group of MS care managers, pharmacists, primary care physicians, and a neurologist specializing in MS.

The survey was divided into 2 sections. In the first section, all participants were given a list of 12 indicators currently used to monitor MS. First, participants were asked to rate each indicator using a 5-point Likert scale from "not important" (1) to "very important" (5). Next, participants were asked to select which 3 indicators were the most meaningful to them. We asked participants this second value-based question to account for a scenario in which most or all indicators were ranked as "important" or "very important" on the Likert scale. An optional text field was included at the end of the value-based questions to allow participants to provide comments or suggestions regarding the indicator list.

In the next section, nonpatient participants were asked to rate the same indicators using a 5-point Likert scale on how feasibly they could be collected in an integrated delivery and finance system where both administrative claims and electronic health record (EHR) data are accessible. Each indicator contained additional information on how it would potentially be measured (via administrative claims and/or EHR).

\section{Survey Rounds}

Participants chose to complete either an online survey through the survey platform SurveyMonkey or a paper survey through U.S. mail. In both rounds, the first page of the survey described the Delphi method and restated the study's purpose. Participants who did not respond received at least 2 reminders via phone or email weekly. After completion of Round 1, descriptive statistics 


\section{FIGURE 1}

Flowchart Diagram of Participants and Response Rates, Delphi Survey Rounds 1 and 2

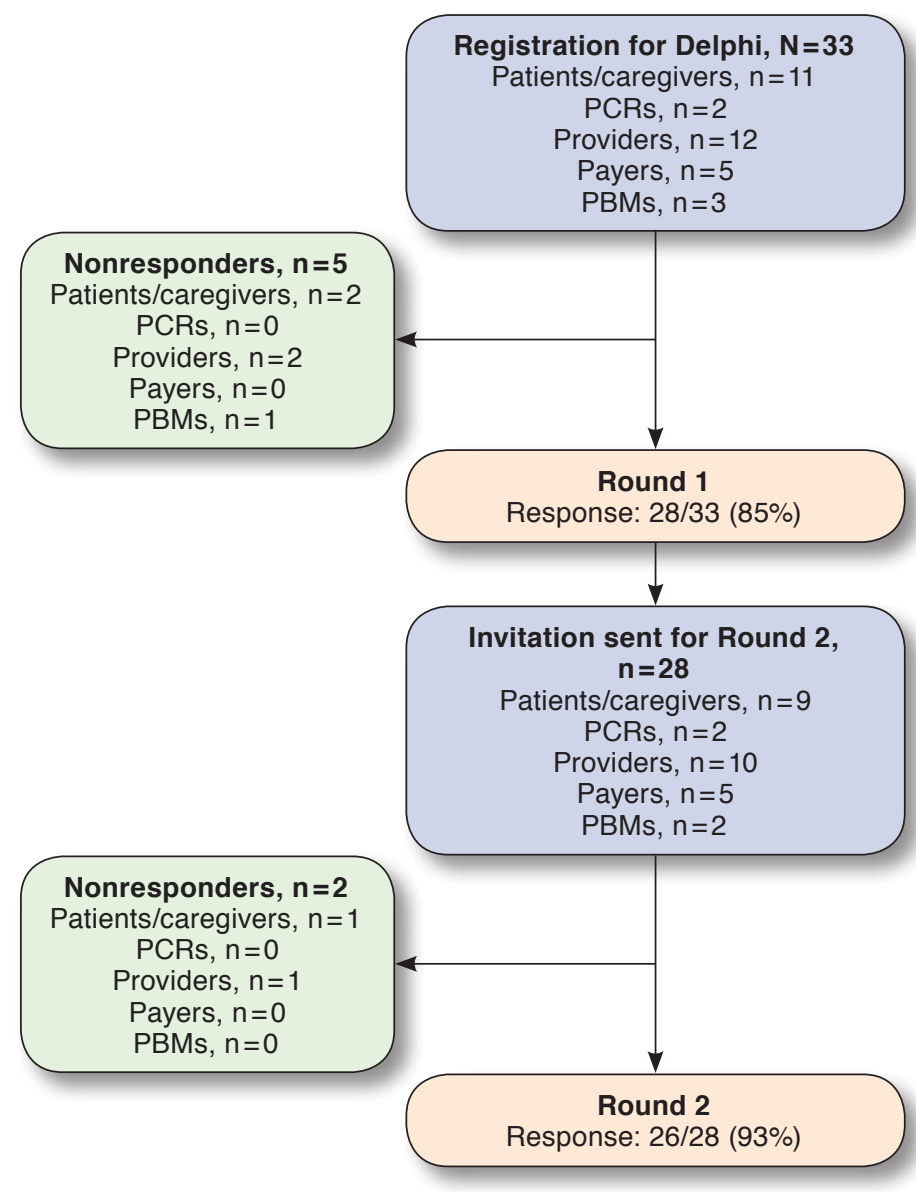

PBMs = pharmaceutical benefits managers; PCRs = pharmaceutical company representatives.

were calculated for each question. The 3 lowest ranked indicators were removed from the Round 2 survey.

The Round 2 survey was individualized to show each participant's Round 1 response alongside the group's average Round 1 rating for each indicator. For example, if a participant rated an indicator as "moderately important" for the Likert scale question (equivalent to a numerical score of 3) and the aggregate mean for that indicator was " 4.50 ," the indicator in Round 2 would have the following text: "Group average from Round 1 was 4.50; Your response was 3." We used a similar approach and comparable language in the remaining questions. Following Delphi methodology, the purpose of this controlled feedback was to inform participants of the group's collective response and provide an opportunity to review and revise their original assessments if desired..$^{13,25}$ Only participants who responded to Round 1 could participate in Round 2. All data were collected between November 2017 and January 2018.

\section{Delphi Panel Recruitment}

The MS Care Management team facilitated recruitment of our patient stakeholders, all of whom were actively participating in the UPMC Health Plan MS Care Management program. We strived to obtain a mix of patients with varied disease severity, and restricted participation to those who had MS for at least 1 year before study onset. During the recruitment phone call, information about the research study was provided; patient questions were answered; and verbal consent was obtained. Patient stakeholders were paid $\$ 20$ for the Round 1 survey and $\$ 30$ for the Round 2 survey; other survey participants were not compensated.

Remaining stakeholders (providers, payers, and pharmaceutical company and pharmacy benefits manager representatives) were selected through convenient sample and were identified as having experience in MS through an internal, multidisciplinary workgroup that included primary care physicians, MS care managers, pharmacists, and a neurologist specializing in MS care. Providers (neurologists, nurses, physician assistants, and specialty pharmacists) were recruited from 3 large, distinct health care systems in southwestern Pennsylvania and were required to have a minimum of 5 years of experience caring for patients with MS. Payer representatives (specialists in Medicaid, Medicare, and commercial product lines) were recruited from regional and government insurers and were involved in clinical and pharmacy operations for patients with MS. Pharmaceutical company representatives were recruited from 2 multinational manufacturers and managed MS medication accounts. Pharmacy benefits manager representatives were from a large, national organization and oversaw contracting for MS medications. Patient and provider stakeholders intentionally constituted the majority of the Delphi panel, since they were considered to have the most direct experience with clinical MS indicators. Additionally, patient and provider viewpoints are often underrepresented in historical value-based contracts between pharmaceutical manufacturers and payers.

\section{Consensus and Statistical Analysis}

Based on previous studies, we defined consensus as $\geq 75 \%$ agreement by stakeholders on the value or feasibility of an indicator (Likert scale scores "important"/"feasible" [4] or "very important"/"very feasible" [5]). ${ }^{14,25}$ A Fisher's exact test was performed to assess differences between patient and nonpatient stakeholder rankings of indicators.

\section{Results}

In total, 33 people were recruited to participate in our modified Delphi survey. From these, 26 stakeholders composed the final panel, where patients/caregivers and providers were almost 


\begin{tabular}{|c|c|c|}
\hline \multirow[b]{2}{*}{ Answer Choices } & \multicolumn{2}{|c|}{ Important (4)/Very Important (5), n (\%) } \\
\hline & Round $1(n=28)$ & Round $2(n=26)$ \\
\hline $\begin{array}{l}\text { Worsening physical disability, meaning decreased ability to move around independently (for patients-this would } \\
\text { mean using assistive devices like wheelchairs and canes) (for providers-this can be measured by disability scales)b }\end{array}$ & $25 \quad(89.3)$ & $24(92.3)$ \\
\hline $\begin{array}{l}\text { Functional impairment (issues with functioning) that disrupts activities of daily living (dressing, bathing, eating, } \\
\text { transferring, toileting independently), as measured by home care, assisted living, and skilled nursing needs }\end{array}$ & $26(92.9)$ & $24(92.3)$ \\
\hline MS flares requiring admission to the hospital b & $23(82.1)$ & $23(88.5)$ \\
\hline Recurrent (repeated) or new spinal cord or brain lesions on imaging studies ${ }^{b}$ & $23(82.1)$ & $22(84.6)$ \\
\hline Medication switches because of failure of treatment, side effects, or other issues with medications ${ }^{b}$ & $22(78.6)$ & $22(84.6)$ \\
\hline Bladder problems, including urinary tract infections and need for urinary catheterization b,c & $21 \quad(77.8)$ & $21 \quad(80.8)$ \\
\hline MS flares requiring multiple steroid courses ${ }^{b}$ & $21 \quad(75.0)$ & $20 \quad(76.9)$ \\
\hline MS flares requiring emergency department visit & $21 \quad(75.0)$ & $19(73.1)$ \\
\hline Worsening mental health issues like depression or anxietyc & $19(70.4)$ & - \\
\hline Need for symptomatic treatments like pain medications or spasticity medications & $19 \quad(67.9)$ & $15 \quad(57.7)$ \\
\hline Missed days of work & $18(64.3)$ & - \\
\hline Fatigue & $16 \quad(57.1)$ & - \\
\hline \multicolumn{3}{|c|}{$\begin{array}{l}\text { aParticipants responded to prompt, "Below is a list of multiple sclerosis (MS) measures currently used to monitor MS. Please rate each of the measures in terms of their } \\
\text { value in providing information about the status of MS." } \\
\text { bConsensus ( } \geq 75 \% \text { agreement) was reached after Round } 2 . \\
\text { 'One participant did not rate this indicator in Round 1, so there were } 27 \text { total responses rather than } 28 . \\
\text { MS = multiple sclerosis. }\end{array}$} \\
\hline
\end{tabular}

equally represented at 8 (31\%) and 9 (35\%) stakeholders, respectively. The remaining panel consisted of 2 (8\%) pharmaceutical company representatives, 5 (19\%) payers, and 2 (8\%) pharmacy benefits manager representatives. Response rates for each round were 28 of 33 (85\%) for Round 1 and 26 of 28 (93\%) for Round 2 (Figure 1). No participants recommended additional indicators to include in the Round 2 survey. "Missed days of work," "fatigue," and "worsening mental health issues like depression or anxiety" received the lowest mean ratings from our panelists and were omitted in Round 2.

\section{Value of Indicators}

After the second Delphi round, consensus was reached on 7 of 9 indicators, reflecting that $\geq 75 \%$ of stakeholders rated these indicators as "important"/"very important" (4 or 5 on the Likert scale; Table 1). The highest-rated indicators were "worsening physical disability, meaning decreased ability to move around independently" and "functional impairment that disrupts activities of daily living, as measured by home care, assisted living, and skilled nursing needs," both achieving 92\% agreement. When asked to select only the top 3 most meaningful indicators, $100 \%$ of participants selected "worsening physical disability, meaning decreased ability to move around independently" (Table 2). "Functional impairment that disrupts activities of daily living, as measured by home care, assisted living, and skilled nursing needs" and "recurrent or new spinal cord or brain lesions on imaging studies" placed second and third at $73 \%$ and $69 \%$ agreement, respectively, although neither reached consensus.
A Fisher's exact test indicated that the only statistically significant difference between patient and nonpatient responses was for "bladder problems including urinary tract infection and need for urinary catheterization," where 38\% of patient stakeholders and none of nonpatient stakeholders selected this indicator as most meaningful ( $P=0.02$, Table 2).

Agreement among all stakeholders increased between Rounds 1 and 2 for the top 3 most meaningful indicators (Table 2): "worsening physical disability" (from 68\% to 100\%); "functional impairment" (from 50\% to 73\%); and "recurrent or new spinal cord or brain lesions on imaging studies" (from $43 \%$ to 69\%). Conversely, agreement on all remaining indicators decreased between Rounds 1 and 2 among all stakeholder groups, most notably: "MS flares requiring emergency department visit" (from 25\% to 15\%); "MS flares requiring multiple steroid courses" (from 21\% to 4\%); "need for symptomatic treatments like pain medications or spasticity medications" (from 11\% to 0\%); and "medication switches because of failure of treatment, side effects, or other issues with medications" (from 25\% to 8\%). Based on Table 2, patients, payers, and pharmacy benefits manager representatives seemed to change their responses for the most valued indicators between Round 1 and Round 2 more than providers and pharmaceutical company representatives.

\section{Feasibility of Indicators}

Half of the MS indicators were rated as "feasible" or "very feasible" to collect by $\geq 75 \%$ of nonpatient stakeholders (Table 3 ). 


\section{TABLE 2 Forced Selection Value-Based Question Results ${ }^{a}$}

\begin{tabular}{|c|c|c|c|c|c|c|c|c|c|c|c|c|}
\hline \multirow[b]{2}{*}{ Answer Choices } & \multicolumn{2}{|c|}{$\begin{array}{l}\text { Patients } \\
\text { n (\%) }\end{array}$} & \multicolumn{2}{|c|}{$\begin{array}{c}\text { Providers } \\
\mathrm{n}(\%)\end{array}$} & \multicolumn{2}{|c|}{$\begin{array}{l}\text { Payers } \\
\text { n (\%) }\end{array}$} & \multicolumn{2}{|c|}{$\begin{array}{l}\text { PCRs } \\
\mathrm{n}(\%)\end{array}$} & \multicolumn{2}{|c|}{$\begin{array}{l}\text { PBM } \\
\text { n (\%) }\end{array}$} & \multicolumn{2}{|c|}{$\begin{array}{l}\text { Total } \\
\text { N (\%) } \\
\end{array}$} \\
\hline & $\begin{array}{c}\mathrm{R} 1 \\
(\mathrm{n}=9)\end{array}$ & $\begin{array}{c}\mathrm{R} 2 \\
(\mathrm{n}=8) \\
\end{array}$ & $\begin{array}{c}\mathrm{R} 1 \\
(\mathrm{n}=10)\end{array}$ & $\begin{array}{c}\mathrm{R} 2 \\
(\mathrm{n}=9) \\
\end{array}$ & $\begin{array}{c}\mathrm{R} 1 \\
(\mathrm{n}=5)\end{array}$ & $\begin{array}{c}\mathrm{R} 2 \\
(\mathrm{n}=5)\end{array}$ & $\begin{array}{c}\mathrm{R} 1 \\
(\mathbf{n}=2)\end{array}$ & $\begin{array}{c}\mathbf{R} 2 \\
(\mathbf{n}=2) \\
\end{array}$ & $\begin{array}{c}\mathrm{Rl} \\
(\mathrm{n}=2)\end{array}$ & $\begin{array}{c}\mathrm{R} 2 \\
(\mathbf{n}=2)\end{array}$ & $\begin{array}{c}\mathrm{R} 1 \\
(\mathrm{n}=28)\end{array}$ & $\begin{array}{c}\mathrm{R} 2 \\
(\mathrm{n}=26) \\
\end{array}$ \\
\hline $\begin{array}{l}\text { Worsening physical disability, meaning decreased ability } \\
\text { to move around independently (for patients, this would } \\
\text { mean using assistive devices like wheelchairs and canes; } \\
\text { for providers, this can be measured by disability scales) }\end{array}$ & $\begin{array}{c}5 \\
(55.6)\end{array}$ & $\begin{array}{c}8 \\
(100.0)\end{array}$ & $\begin{array}{c}9 \\
(90.0)\end{array}$ & $\begin{array}{c}9 \\
(100.0)\end{array}$ & $\begin{array}{c}3 \\
(60.0)\end{array}$ & $\begin{array}{c}5 \\
(100.0)\end{array}$ & $\begin{array}{c}2 \\
(100.0)\end{array}$ & $\begin{array}{c}2 \\
(100.0)\end{array}$ & $\begin{array}{c}0 \\
(0)\end{array}$ & $\begin{array}{c}2 \\
(100.0)\end{array}$ & $\begin{array}{c}19 \\
(67.9)\end{array}$ & $\begin{array}{c}26 \\
(100.0)\end{array}$ \\
\hline $\begin{array}{l}\text { Functional impairment (issues with functioning) that } \\
\text { disrupts activities of daily living (dressing, bathing, eat- } \\
\text { ing, transferring, toileting independently), as measured by } \\
\text { home care, assisted living, and skilled nursing needs }\end{array}$ & $\begin{array}{c}6 \\
(66.7)\end{array}$ & $\begin{array}{c}7 \\
(87.5)\end{array}$ & $\begin{array}{c}4 \\
(40.0)\end{array}$ & $\begin{array}{c}5 \\
(55.6)\end{array}$ & $\begin{array}{c}3 \\
(60.0)\end{array}$ & $\begin{array}{c}4 \\
(80.0)\end{array}$ & $\begin{array}{c}1 \\
(50.0)\end{array}$ & $\begin{array}{c}2 \\
(100.0)\end{array}$ & $\begin{array}{c}0 \\
(0)\end{array}$ & $\begin{array}{c}1 \\
(50)\end{array}$ & $\begin{array}{c}14 \\
(50.0)\end{array}$ & $\begin{array}{c}19 \\
(73.1)\end{array}$ \\
\hline $\begin{array}{l}\text { Recurrent (repeated) or new spinal cord or brain lesions } \\
\text { on imaging studies }\end{array}$ & $\begin{array}{c}3 \\
(33.3) \\
\end{array}$ & $\begin{array}{c}5 \\
(62.5) \\
\end{array}$ & $\begin{array}{c}7 \\
(70.0) \\
\end{array}$ & $\begin{array}{c}7 \\
(77.8)\end{array}$ & $\begin{array}{c}0 \\
(0)\end{array}$ & $\begin{array}{c}2 \\
(40.0)\end{array}$ & $\begin{array}{c}1 \\
(50.0)\end{array}$ & $\begin{array}{c}2 \\
(100.0)\end{array}$ & $\begin{array}{c}1 \\
(50.0)\end{array}$ & $\begin{array}{c}2 \\
(100.0) \\
\end{array}$ & $\begin{array}{c}12 \\
(42.9)\end{array}$ & $\begin{array}{c}18 \\
(69.2) \\
\end{array}$ \\
\hline MS flares requiring admission to the hospital & $\begin{array}{c}1 \\
(11.1)\end{array}$ & $\begin{array}{c}0 \\
(0)\end{array}$ & $\begin{array}{c}1 \\
(10.0) \\
\end{array}$ & $\begin{array}{c}3 \\
(33.3) \\
\end{array}$ & $\begin{array}{c}2 \\
(40.0) \\
\end{array}$ & $\begin{array}{c}1 \\
(20.0) \\
\end{array}$ & $\begin{array}{c}0 \\
(0)\end{array}$ & $\begin{array}{c}0 \\
(0)\end{array}$ & \begin{tabular}{c|c}
2 \\
$(100.0)$
\end{tabular} & $\begin{array}{c}1 \\
(50.0) \\
\end{array}$ & $\begin{array}{c}6 \\
(21.4) \\
\end{array}$ & $\begin{array}{c}5 \\
(19.2) \\
\end{array}$ \\
\hline MS flares requiring emergency department visit & $\begin{array}{c}1 \\
(11.1)\end{array}$ & $\begin{array}{c}0 \\
(0)\end{array}$ & $\begin{array}{c}1 \\
(10.0)\end{array}$ & $\begin{array}{c}1 \\
(11.1)\end{array}$ & $\begin{array}{c}3 \\
(60.0)\end{array}$ & $\begin{array}{c}3 \\
(60.0) \\
\end{array}$ & $\begin{array}{c}0 \\
(0)\end{array}$ & $\begin{array}{c}0 \\
(0)\end{array}$ & \begin{tabular}{c|c}
2 \\
$(100.0)$
\end{tabular} & $\begin{array}{c}0 \\
(0)\end{array}$ & $\begin{array}{c}7 \\
(25.0)\end{array}$ & $\begin{array}{c}4 \\
(15.4)\end{array}$ \\
\hline $\begin{array}{l}\text { Bladder problems, including urinary tract infections and } \\
\text { need for urinary catheterizationc }\end{array}$ & $\begin{array}{c}2 \\
(22.2)\end{array}$ & $\begin{array}{c}3 \\
(37.5)\end{array}$ & $\begin{array}{c}0 \\
(0)\end{array}$ & $\begin{array}{c}0 \\
(0)\end{array}$ & $\begin{array}{c}2 \\
(40.0) \\
\end{array}$ & $\begin{array}{c}0 \\
(0)\end{array}$ & $\begin{array}{c}0 \\
(0)\end{array}$ & $\begin{array}{c}0 \\
(0)\end{array}$ & $\begin{array}{c}0 \\
(0)\end{array}$ & $\begin{array}{c}0 \\
(0)\end{array}$ & $\begin{array}{c}4 \\
(14.3)\end{array}$ & $\begin{array}{c}3 \\
(11.5)\end{array}$ \\
\hline $\begin{array}{l}\text { Medication switches because of failure of treatment, side } \\
\text { effects, or other issues with medications }\end{array}$ & $\begin{array}{c}2 \\
(22.2)\end{array}$ & $\begin{array}{c}1 \\
(12.5)\end{array}$ & $\begin{array}{c}2 \\
(20.0) \\
\end{array}$ & $\begin{array}{c}1 \\
(11.1)\end{array}$ & $\begin{array}{c}1 \\
(20.0)\end{array}$ & $\begin{array}{c}0 \\
(0)\end{array}$ & $\begin{array}{c}2 \\
(100.0)\end{array}$ & $\begin{array}{c}0 \\
(0)\end{array}$ & $\begin{array}{c}0 \\
(0)\end{array}$ & $\begin{array}{c}0 \\
0 \\
(0)\end{array}$ & $\begin{array}{c}7 \\
(25.0)\end{array}$ & $\begin{array}{c}2 \\
(7.7) \\
\end{array}$ \\
\hline MS flares requiring multiple steroid courses & $\begin{array}{c}1 \\
(11.1)\end{array}$ & $\begin{array}{c}0 \\
0 \\
(0)\end{array}$ & $\begin{array}{c}5 \\
(50.0) \\
\end{array}$ & $\begin{array}{c}1 \\
(11.1)\end{array}$ & $\begin{array}{c}0 \\
(0)\end{array}$ & $\begin{array}{c}0 \\
(0)\end{array}$ & $\begin{array}{c}0 \\
0 \\
(0)\end{array}$ & $\begin{array}{c}0 \\
(0)\end{array}$ & $\begin{array}{c}0 \\
0 \\
(0)\end{array}$ & $\begin{array}{c}0 \\
0 \\
(0)\end{array}$ & $\begin{array}{c}6 \\
(21.4) \\
\end{array}$ & $\begin{array}{c}1 \\
(3.8)\end{array}$ \\
\hline $\begin{array}{l}\text { Need for symptomatic treatments like pain medications } \\
\text { or spasticity medications }\end{array}$ & $\begin{array}{c}3 \\
(33.3) \\
\end{array}$ & $\begin{array}{c}0 \\
0 \\
(0)\end{array}$ & $\begin{array}{c}0 \\
(0)\end{array}$ & $\begin{array}{c}0 \\
(0)\end{array}$ & $\begin{array}{c}0 \\
(0)\end{array}$ & $\begin{array}{c}0 \\
0 \\
(0)\end{array}$ & $\begin{array}{c}0 \\
0 \\
(0)\end{array}$ & $\begin{array}{c}0 \\
(0)\end{array}$ & $\begin{array}{c}0 \\
(0)\end{array}$ & $\begin{array}{c}0 \\
(0)\end{array}$ & $\begin{array}{c}3 \\
(10.7)\end{array}$ & $\begin{array}{c}0 \\
0 \\
(0)\end{array}$ \\
\hline $\begin{array}{l}\text { Worsening mental health issues like depression } \\
\text { or anxiety }\end{array}$ & $\begin{array}{c}1 \\
(11.1)\end{array}$ & - & $\begin{array}{c}1 \\
(10.0)\end{array}$ & - & $\begin{array}{c}0 \\
(0)\end{array}$ & - & $\begin{array}{c}0 \\
(0)\end{array}$ & - & $\begin{array}{c}0 \\
(0)\end{array}$ & - & $\begin{array}{c}2 \\
(7.2)\end{array}$ & - \\
\hline Missed days of work & $\begin{array}{c}0 \\
(0)\end{array}$ & - & $\begin{array}{c}0 \\
0 \\
(0)\end{array}$ & - & $\begin{array}{c}1 \\
(20.0)\end{array}$ & - & $\begin{array}{c}0 \\
0 \\
(0)\end{array}$ & - & $\begin{array}{c}1 \\
(50.0)\end{array}$ & - & $\begin{array}{c}2 \\
(7.2)\end{array}$ & - \\
\hline Fatigue & $\begin{array}{c}2 \\
(22.2)\end{array}$ & - & $\begin{array}{c}0 \\
(0)\end{array}$ & - & $\begin{array}{c}0 \\
(0)\end{array}$ & - & $\begin{array}{c}0 \\
(0)\end{array}$ & - & $\begin{array}{c}0 \\
(0)\end{array}$ & - & $\begin{array}{c}2 \\
(7.2)\end{array}$ & - \\
\hline
\end{tabular}

aParticipants responded to the prompt, "If we had to limit the number of MS measures to 3 that are most meaningful, which ones would you choose to keep (please select only 3)?"

${ }^{b}$ Consensus ( $\geq 75 \%$ agreement) was reached after Round 2

'Statistically significant difference found between patient and nonpatient stakeholder groups; $P=0.02$ for the indicator noted.

MS = multiple sclerosis; PBMs = pharmacy benefits managers; PCRs = pharmaceutical company representatives

"MS flares requiring an emergency department visit" and "MS flares requiring inpatient admission" were rated as the most feasibly collected, both receiving $89 \%$ agreement. "Medication switches because of failure of treatment, side effects, or other issues with medications" and "MS flares requiring multiple steroid courses" received 83\% agreement each, followed by "need for symptomatic treatments like pain medications or spasticity medications" at $78 \%$ agreement. "Worsening physical disability, as measured by disability scales;" "worsening physical disability as measured by use of assistive devices like wheelchairs and canes;" and "functional impairment that disrupts activities of daily living, as measured by home care, assisted living, and skilled nursing needs" were the lowest rated indicators at 44\%, $33 \%$, and 22\%, respectively. Results were consistent between Rounds 1 and 2 .

\section{Discussion}

Through use of the Delphi method, this study uniquely involved the views of a range of stakeholders to explore which MS clinical indicators are the most meaningful and feasibly collected for a value-based contract. To our knowledge, there are no published studies where the Delphi method has been used to inform value-based contracts. ${ }^{10}$ Unanimously, "worsening physical disability" was agreed upon as the most meaningful MS indicator. Conversely, "MS flares requiring emergency department visits" and "MS flares requiring inpatient admissions" were considered the most feasibly collected indicators, and "worsening physical disability" was 1 of the lowest rated with regards to feasibility of data collection.

The Delphi method's strength in producing consensus is demonstrated by changes in participants' responses from Round 1 to Round 2. For instance, in Round 1, when participants were 
TABLE 3 Likert Scale Feasibility Question Results ${ }^{a}$

Feasible (4)/ Very Feasible (5), n (\%)

Answer Choices

Round 1 Round 2

$(\mathrm{n}=19) \quad(\mathrm{n}=18)$

MS flares requiring emergency department visit

(measured through claims)

MS flares requiring inpatient admission (measured through claims) ${ }^{\mathrm{b}}$

Medication switches because of failure of treatment, side effects, or other issues with medications (measured through claims) ${ }^{\mathrm{b}}$

MS flares requiring multiple steroid courses (measured through pharmaceutical claims) $^{\mathrm{b}}$

Need for symptomatic treatments like pain medications or spasticity medications (measured through claims or EHR) ${ }^{b}$

Bladder dysfunction, as measured by frequency of urinary tract infections and need for urinary catheterization (measured through claims or EHR)

Recurrent or new spinal cord or brain lesions (measured through claims or EHR)

$18(94.7) \quad 16(88.9)$

Worsening physical disability, as measured by disability scales like Expanded Disability Status Scale, Patient-Determined Disease Steps, or Multiple Sclerosis Rating Scale, Revised (measured through EHR)

Worsening physical disability, as measured by use of assistive devices, such aswheelchairs and canes (measured through claims)

Functional impairment that disrupts activities of daily living (dressing, bathing, eating, transferring, toileting independently), as measured by home care, assisted living, and skilled nursing needs (measured through claims or EHR)

Missed days of work

Worsening mental health issues like depression or anxiety

Fatigue

a Nonpatient participants responded to prompt, "Please rate each measure below on how feasible they are to collect in an integrated delivery and finance system where both administrative claims and EHR data are accessible."

${ }^{b}$ Consensus (greater than or equal to $75 \%$ agreement) was reached after Round 2. $E H R=$ electronic health record; $M S=$ multiple sclerosis .

asked to rank which indicator was the most meaningful to them, $21 \%$ of participants selected "MS flares requiring multiple steroid courses" as a top meaningful indicator. In Round 2, however, just $4 \%$ of panelists selected this indicator, which was likely influenced by the controlled feedback and aggregation of group responses provided. Through the Delphi method, participants are empowered to use the group's viewpoints as additional information in evaluating their original assessments and in informing participants' decisions to maintain their previous round responses or to change any of their responses based on the group's feedback. This method achieves the ultimate aim of reaching a collective consensus among a large, diverse group of stakeholders. Of interest, we observed that patients, payers, and pharmacy benefits manager representatives changed their responses for the most valued indicators from Round 1 to Round 2 more so than provider and pharmaceutical company representative stakeholders. This difference suggests potential social desirability bias where different groups may be more or less likely to be influenced by the group's aggregate response from previous rounds. To our knowledge, previous Delphi studies have not focused on which types of stakeholders might be more or less sensitive to modifying their responses based on controlled feedback.

Previous value-based contracts for MS have selected measures which can only be derived from claims and EHR data. These contracts included MS-related emergency department visits, number of hospitalizations, medication adherence, and relapse rates. ${ }^{10}$ In our study, when panelists were asked to select which 3 indicators were the most meaningful, "MS flares requiring an emergency department visit" only received 15\% agreement, while "MS flares requiring admission to the hospital" received 19\% agreement. Our results therefore identify an important challenge with commonly used MS indicators, especially those in publicized value-based contracts: Because most value-based contracts are based on administrative claims or EHR data, the most feasibly collected indicators may not be the most meaningful, and the most meaningful indicators may not be feasibly collected.

Our findings suggest that MS-based initiatives and research programs should improve their ability to assess and report meaningful patient-reported outcomes such as disability and function and present us with an innovative opportunity to understand how to operationalize the inclusion of patientreported outcomes in a value-based contract. For instance, because disability is difficult to measure with claims or EHR data, one could consider collecting these data through validated patient-reported measures, such as Guy's Neurological Disability Scale, Patient Determined Disease Steps, or the Multiple Sclerosis Impact Scale-29.17,24,26,27

To our knowledge, although other studies have used Delphi methodology to understand important MS outcomes, none have used the Delphi method to answer a business need such as the development of a value-based contract. For instance, a portfolio of studies used Delphi methodology to understand which MS outcomes should be in the International Classification of Functioning Core Set for MS. These studies administered Delphi surveys to physicians, occupational therapists, physical therapists, and speech and language therapists separately to assess these providers' perspectives on the most significant functional and environmental challenges for patients living with MS. This set of Delphi studies identified body functions 
and body structures (i.e., muscle tone, involuntary movement reaction, and gait pattern functions) and activities and participation (i.e., changing basic body position, transferring oneself, and lifting and carrying objects) outcomes as important intervention categories in the Core Set for MS. ${ }^{28-33}$ Importantly, in contrast with our approach, none of these Delphi studies captured patient, payer, or pharmacy viewpoints.

Another Delphi study was conducted to identify which quality indicators could appropriately be used to gauge care delivery for persons living with MS. ${ }^{34}$ Comparable with our study, a 2-round Delphi survey was conducted with a multidisciplinary panel (MS patients, directors of MS patient advocacy organizations, neurologists, rehabilitation physicians, nurses, therapists, and health care administrators), although payers and pharmaceutical company and pharmacy benefits manager representatives were not present. In contrast to our study, the study team first surveyed which care domains were valued by panelists and then selected MS indicators that measured only the highly rated domains. The 15-18 Delphi panelists identified the following domains/indicators as the most highly valued: bladder dysfunction, use of disease-modifying agents, management of exacerbations, difficulties with activities of daily living, and general preventive care. The study team further categorized which indicators would be suitable for use as a quality measure; 1 criterion for indicator validity was the ability to be measured through administrative claims, whereas we deemed indicators as valid regardless of their ability to be measured through conventional means.

\section{Limitations}

Our study has limitations to consider. First, we sampled our patient population through our MS care management program, possibly introducing selection bias in our patient stakeholder group because it may not be representative of a broader MS patient population. However, the directional effect of this possible selection bias is uncertain: On one hand, the care management program receives referrals from neurologists and other providers for patients who have been identified as needing additional service coordination or disease management, indicating that this cohort could have poorer disease control and more barriers to care than the general MS population. On the other hand, patients engaged in the care management program who responded to our survey may have higher patient activation levels than others. Nevertheless, we attempted to account for potential bias by including patients with varying MS experiences, such as duration of disease, symptoms, severity, and use of medications.

Second, our patient and provider stakeholders were mostly from a single geographic region-southwestern Pennsylvaniaand their opinions may not be representative of a broader population of MS patients and providers. Importantly, we strived to include different types of providers (neurologists, nurses, physician assistants, and specialty pharmacists) who might have varying experiences with these MS indicators.

Third, while we included a payer stakeholder group, we did not account for any employer group representation, which is a stakeholder group that might be particularly interested in MS outcomes. Fourth, our response rates were 85\% and 93\% for Rounds 1 and 2, respectively; although these were not 100\%, we found that they were consistent with response rates found in the literature and unlikely to produce meaningful bias. ${ }^{13}$

Finally, our panel included less representation from nonpatient, nonprovider stakeholders. It is possible that if we had surveyed larger groups of individuals in each stakeholder group, our findings may have changed. Nevertheless, we intentionally oversampled patients and providers in our panel, and our Delphi panel size was similar to, if not larger than, other published Delphi studies in MS. 29,35,36

\section{Conclusions}

Through our study's novel use of the Delphi method, we identified meaningful and feasibly collected MS indicators for diverse stakeholders. We found that indicators that are perceived as most meaningful such as disability and functional impairment are not considered feasibly collected through conventional means. The essence of value-based contracting is to incentivize improved patient outcomes by linking payment to performance. Our results should inform the development of innovative value-based contracts for MS medications that incorporate important patient outcomes relevant to multiple stakeholders.

\section{Authors}

ELIZABETH C.S. SWART, BS; LYNN M. NEILSON, PhD; and WILLIAM H. SHRANK, MD, MSHS, Center for Value-Based Pharmacy Initiatives, UPMC Health Plan, Pittsburgh, Pennsylvania. CHESTER B. GOOD, MD, MPH, and NATASHA PAREKH, MD, MS, Center for Value-Based Pharmacy Initiatives, UPMC Health Plan, Pittsburgh, Pennsylvania, and Division of General Internal Medicine, University of Pittsburgh. ROCHELLE HENDERSON, $\mathrm{PhD}$, Express Scripts Holding Company, St. Louis, Missouri, and CHRONIS MANOLIS, RPh, Center for Value-Based Pharmacy Initiatives and Pharmacy Division, UPMC Health Plan, Pittsburgh, Pennsylvania.

AUTHOR CORRESPONDENCE: Natasha Parekh, MD, MS, Center for Value-Based Pharmacy Initiatives, UPMC Health Plan, 600 Grant St., Pittsburgh, PA 15219. Tel.: 412.454.1325;

E-mail: natashaparekh1@gmail.com 


\section{DISCLOSURES}

This study was supported by a grant from Express Scripts Holding Company, which provided research funding to the UPMC Center for Value-Based Pharmacy Initiatives for work on this study. Swart, Neilson, Good, and Parekh are employed by the UPMC Center for Value-Based Pharmacy Initiatives. Manolis is the Chief Pharmacy Officer of UPMC Health Plan, and Shrank was the Chief Medical Officer of UPMC Insurance Services Division at the time of this study. Henderson is employed by Express Scripts Holding Company.

\section{REFERENCES}

1. Maroney M, Hunter SF. Implications for multiple sclerosis in the era of the Affordable Care Act: a clinical overview. Am J Manag Care. 2014;20 (11 Suppl):S220-27.

2. Dashputre AA, Kamal KM, Pawar G. Cost-effectiveness of peginterferon beta-la and alemtuzumab in relapsing-remitting multiple sclerosis. J Manag Care Spec Pharm. 2017;23(6):666-76. Available at: https://www.jmcp.org/ doi/10.18553/jmcp.2017.23.6.666

3. McDonald WI, Compston A, Edan G, et al. Recommended diagnostic criteria for multiple sclerosis: guidelines from the International Panel on the Diagnosis of Multiple Sclerosis. Ann Neurol. 2001;50(1):121-27.

4. Owens GM. Economic burden of multiple sclerosis and the role of managed care organizations in multiple sclerosis management. Am J Manag Care. 2016;22(6 Suppl):S151-58

5. Hartung DM. Economics and cost-effectiveness of multiple sclerosis therapies in the USA. Neurotherapeutics. 2017;14(4):1018-26.

6. Express Scripts. 2017 drug trend report. February 2018. Available at: http://lab.express-scripts.com/lab/drug-trend-report/previous-reports. Accessed May 21, 2019.

7. Hartung DM, Bourdette DN, Ahmed SM, Whitham RH. The cost of multiple sclerosis drugs in the U.S. and the pharmaceutical industry: too big to fail? Neurology. 2015;84(21):2185-92.

8. AMCP Partnership Forum: advancing value-based contracting. J Manag Care Spec Pharm. 2017;23(11):1096-102. Available at: https://www.jmcp.org/ doi/10.18553/jmcp.2017.17342.

9. Carlson JJ, Sullivan SD, Garrison LP, Neumann PJ, Veenstra DL. Linking payment to health outcomes: a taxonomy and examination of performancebased reimbursement schemes between healthcare payers and manufacturers. Health Policy. 2010;96(3):179-90

10. Yu JS, Chin L, Oh J, Farias J. Performance-based risk-sharing arrangements for pharmaceutical products in the United States: a systematic review. J Manag Care Spec Pharm. 2017;23(10):1028-40. Available at: https://www. jmcp.org/doi/10.18553/jmcp.2017.23.10.1028.

11. Gentilini P, Bernardi M, Bolondi L, et al. The rational use of albumin in patients with cirrhosis and ascites: a Delphi study for the attainment of a consensus on prescribing standards. Dig Liver Dis. 2004;36(8):539-46.

12. Eubank BH, Mohtadi NG, Lafave MR, et al. Using the modified Delphi method to establish clinical consensus for the diagnosis and treatment of patients with rotator cuff pathology. BMC Med Res Methodol. 2016;16(1):56

13. Boulkedid R, Abdoul H, Loustau M, Sibony O, Alberti C. Using and reporting the Delphi method for selecting healthcare quality indicators: a systematic review. PloS One. 2011;6(6):e20476.

14. Hsu C, Sandford BA. The Delphi technique: making sense of consensus. Pract Assess Res Eval. 2007;12(10):1-8.

15. Meyer-Moock S, Feng YS, Maeurer M, Dippel FW, Kohlmann T Systematic literature review and validity evaluation of the Expanded Disability Status Scale (EDSS) and the Multiple Sclerosis Functional Composite (MSFC) in patients with multiple sclerosis. BMC Neurology. 2014:14(1):58.
16. Foley JF, Brandes DW. Redefining functionality and treatment efficacy in multiple sclerosis. Neurology. 2009;72(23 Suppl 5):S1-11.

17. Amato MP, Portaccio E. Clinical outcome measures in multiple sclerosis. J Neurol Sci. 2007;259(1-2):118-22.

18. Daumer M, Neuhaus A, Herbert J, Ebers G. Prognosis of the individual course of disease: the elements of time, heterogeneity and precision. J Neurol Sci. 2009;287(Suppl 1):S50-55

19. Rudick RA, Miller DM. Health-related quality of life in multiple sclerosis current evidence, measurement and effects of disease severity and treatment. CNS Drugs. 2008;22(10):827-39.

20. Sharrack B, Hughes RA, Soudain S, Dunn G. The psychometric properties of clinical rating scales used in multiple sclerosis. Brain. 1999;122(1):141-59.

21. Sipe JC, Knobler RL, Braheny SL, Rice GP, Panitch HS, Oldstone MB. A neurologic rating scale (NRS) for use in multiple sclerosis. Neurology. 1984:34(10):1368-72

22. Vickrey BG. Multiple Sclerosis Quality of Life (MSQOL)-54 Instrument. 1995. Available at: https://www.nationalmssociety.org/NationalMSSociety/media/ MSNationalFiles/Brochures/MSQOL54_995.pdf. Accessed May 21, 2019.

23. Riazi A. Patient-reported outcome measures in multiple sclerosis. Int MS J. 2006;13(3):92-99.

24. Cohen BA, Khan O, Jeffery DR, et al. Identifying and treating patients with suboptimal responses. Neurology. 2004;63(12 Suppl 6):S33-40.

25. Diamond IR, Grant RC, Feldman BM, et al. Defining consensus: a systematic review recommends methodologic criteria for reporting of Delphi studies. J Clin Epidemiol. 2014;67(4):401-09.

26. Sharrack B, Hughes RA. The Guy's Neurological Disability Scale (GNDS): a new disability measure for multiple sclerosis. Mult Scler. 1999;5(4):223-33

27. Learmonth YC, Motl RW, Sandroff BM, Pula JH, Cadavid D. Validation of patient determined disease steps (PDDS) scale scores in persons with multiple sclerosis. BMC Neurology. 2013;13(1):37.

28. Coenen M, Cieza A, Freeman J, et al; Members of the Consensus Conference. The development of ICF Core Sets for multiple sclerosis: results of the International Consensus Conference. J Neurol. 2011;258(8):1477-88.

29. Khan F, Pallant JF. Use of the International Classification of Functioning, Disability and Health (ICF) to identify preliminary comprehensive and brief core sets for multiple sclerosis. Disabil Rehabil. 2007;29(3):205-13.

30. Berno S, Coenen M, Leib A, Cieza A, Kesselring J. Validation of the Comprehensive International Classification of Functioning, Disability, and Health Core Set for multiple sclerosis from the perspective of physicians. J Neurol. 2012;259(8):1713-26.

31. Conrad A, Coenen M, Schmalz H, et al. Validation of the Comprehensive ICF Core Set for Multiple Ssclerosis from the perspective of physical therapists. Phys Ther. 2012;92(6):799-820.

32. Conrad A, Coenen M, Schmalz H, Kesselring J, Cieza A. Validation of the Comprehensive ICF Core Set for Multiple Sclerosis from the perspective of occupational therapists. Scand J Occup Ther. 2012;19(6):468-87.

33. Renom M, Conrad A, Bascuñana $\mathrm{H}$, et al. Content validity of the Comprehensive ICF Core Set for Multiple Sclerosis from the perspective of speech and language therapists. Int J Lang Commun Disord. 2014;49(6):672-86.

34. Cheng EM, Crandall CJ, Bever Jr CT, et al. Quality indicators for multiple sclerosis. Mult Scler J. 2010;16(8):970-80.

35. Potter K, Cohen ET, Allen DD, et al. Outcome measures for individuals with multiple sclerosis: recommendations from the American Physical Therapy Association Neurology Section Task Force. Phys Ther. 2014:94(5):593-608.

36. Hobart J, Cano S, Baron R, et al. Achieving valid patient-reported outcomes measurement: a lesson from fatigue in multiple sclerosis. Mult Scler J. 2013;19(13):1773-83. 
The purpose of this research is to evaluate multiple sclerosis (MS) measures currently used to monitor MS and to understand which measures are the most meaningful and valuable to patients living with MS. We will be consulting a panel of people considered to be experts on MS, and will ask them to complete a brief online survey (below). This survey will use the Delphi Method, where experts answer questionnaires in two or more rounds. If you are willing to participate, the survey will ask about different MS measures (for example, MS flares and functional status) and how different measures are perceived by the participant.

1. Are you a patient, caregiver, provider, payer, pharmaceutical company representative, or pharmacy benefits manager representative (Please select one)?

Patient

Caregiver

Provider
Payer

Pharmaceutical Company Representative

Pharmacy Benefits Manager Representative

2. Below is a list of multiple sclerosis (MS) measures currently used to monitor MS. Please rate each of the measures in terms of their value in providing information about the status of MS.

\title{
Slightly Important Moderately \\ Not Important (1) $\quad$ (2) $\quad$ Important (3) $\quad$ Important (4) Very Important (5)
}

\begin{abstract}
MS flares requiring
emergency

department visit

MS flares requiring

admission to the

hospital

MS flares requiring

multiple steroid

courses
\end{abstract}

Worsening physical

disability, meaning

decreased ability to

move around

independently (for

patients, this would

mean using assistive

devices like

wheelchairs and

canes; for providers,

this can be measured

by disability scales) 
Need for symptomatic treatments like pain medications or spasticity medications

Bladder problems, including urinary tract infections and need for urinary catheterization

Medication switches because of failure of treatment, side effects, or other issues with medications

Functional impairment (issues with functioning) that disrupts activities of daily living (dressing, bathing, eating, transferring, toileting independently), as measured by home care, assisted living, and skilled nursing needs

\section{Recurrent (repeated) or new spinal cord or brain lesions on imaging studies}

Missed days of work

Fatigue

Worsening mental health issues like depression or anxiety 


\section{* 3. If we had to limit the number of MS measures to 3 that are most meaningful, which ones would you choose to keep (please select only 3 )?}

Worsening physical disability, meaning decreased ability to move around independently (for patients-this would mean using assistive devices like wheelchairs and canes) (for providers-this can be measured by disability scales)

Functional impairment (issues with functioning) that disrupts activities of daily living (dressing, bathing, eating, transferring, toileting independently), as measured by home care, assisted living, and skilled nursing needs

Need for symptomatic treatments like pain medications or spasticity medications

Bladder problems, including urinary tract infections and need for urinary catheterization

Medication switches because of failure of treatment, side effects, or other issues with medications

Missed days of work

Fatigue

Worsening mental health issues like depression or anxiety on imaging studies MS flares requiring emergency department visit

MS flares requiring admission to the hospital

MS flares requiring multiple steroid courses

\section{Are there any important measures for MS that we are missing?}

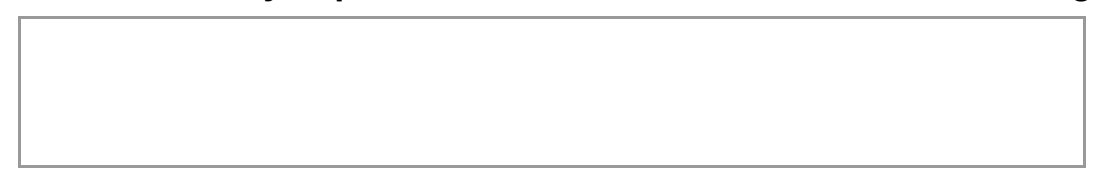


5. Please rate each measure below on how feasible they are to collect in an integrated delivery and finance system where both administrative claims and electronic health record (EHR) data are accessible.

Not At All Feasible

(1)

MS flares requiring

emergency

department visit

(measured through

claims)

MS flares requiring inpatient admission (measured through claims)

MS flares requiring multiple steroid courses (measured through pharmaceutical claims)

Worsening physical disability, as measured by use of assistive devices like wheelchairs and canes (measured through claims)

Worsening physical disability, as measured by disability scales like Expanded Disability Status Scale, PatientDetermined Disease Steps, or Multiple Sclerosis Rating Scale, Revised (measured through EHR)

\section{sible}

Moderately

Feasible (3)
Feasible (4)
Very Feasible (5) 
Not At All Feasible

(1)

Need for symptomatic

treatments like pain

medications or

spasticity medications

(measured through

claims or EHR)

Bladder dysfunction, as measured by

frequency of urinary tract infections and need for urinary catheterization (measured through claims or EHR)

Medication switches because of failure of treatment, side effects, or other issues with medications (measured through claims)

Functional impairment that disrupts activities of daily living (dressing, bathing, eating, transferring, toileting independently), as measured by home care, assisted living, and skilled nursing needs (measured through claims or EHR)

s

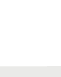

,

Moderately

Feasible (3)
(2)

easible
Feasible (4)

Very Feasible (5)

O


APPENDIX Modified Delphi Survey: Round 1 (continued)

Not At All Feasible Slightly Feasible

(1)

(2)

Recurrent or new spinal cord or brain lesions (measured through claims or EHR)

Missed days of work (measured through EHR)

Fatigue (measured through claims or EHR)

Worsening mental health issues like depression or anxiety (measured through claims or EHR)
Moderately

Feasible (3)

Feasible (4) Very Feasible (5) 